\title{
Modelling the Material Resistance of Wood-Part 2: Validation and Optimization of the Meyer-Veltrup Model
}

\author{
Christian Brischke ${ }^{1, *(D)}$, Gry Alfredsen ${ }^{2}\left(\mathbb{D}\right.$, Miha Humar $^{3}\left(\mathbb{D}\right.$, Elena Conti ${ }^{4}\left(\mathrm{D}\right.$, Laurie Cookson ${ }^{5}$, \\ Lukas Emmerich ${ }^{1}$ D , Per Otto Flæte ${ }^{6}$, Stefania Fortino ${ }^{7}$, Lesley Francis ${ }^{8}$, Ulrich Hundhausen ${ }^{6}$, Ilze Irbe ${ }^{9}$ (D), \\ Kordula Jacobs ${ }^{10}$, Morten Klamer ${ }^{11}$, Davor Kržišnik ${ }^{3}{ }^{1}$, Boštjan Lesar ${ }^{3}$ (D) Eckhard Melcher ${ }^{12}$, \\ Linda Meyer-Veltrup ${ }^{13}$, Jeffrey J. Morrel1 ${ }^{14}$, Jack Norton ${ }^{8}$, Sabrina Palanti ${ }^{15} \mathbb{D}$, Gerald Presley ${ }^{16}$, \\ Ladislav Reinprecht ${ }^{17}$ (D), Tripti Singh ${ }^{18}\left(\mathbb{D}\right.$, Rod Stirling ${ }^{19}$ (D), Martti Venäläinen ${ }^{20}$, Mats Westin ${ }^{21}$, \\ Andrew H. H. Wong ${ }^{22}$ and Ed Suttie 23
}

check for updates

Citation: Brischke, C.; Alfredsen, G.; Humar, M.; Conti, E.; Cookson, L.; Emmerich, L.; Flæte, P.O.; Fortino, S.; Francis, L.; Hundhausen, U.; et al. Modelling the Material Resistance of Wood-Part 2: Validation and Optimization of the Meyer-Veltrup Model. Forests 2021, 12, 576. https:// doi.org/10.3390/f12050576

Academic Editor: Angela Lo Monaco

Received: 29 March 2021

Accepted: 16 April 2021

Published: 6 May 2021

Publisher's Note: MDPI stays neutral with regard to jurisdictional claims in published maps and institutional affiliations.

Copyright: (c) 2021 by the authors. Licensee MDPI, Basel, Switzerland. This article is an open access article distributed under the terms and conditions of the Creative Commons Attribution (CC BY) license (https:// creativecommons.org/licenses/by/ $4.0 /)$.
1 Wood Biology and Wood Products, University of Goettingen, 37077 Goettingen, Germany; lukas.emmerich@uni-goettingen.de

2 Norwegian Institute of Bioeconomy Research (NIBIO), Division of Forests and Forest Resources, Wood Technology, 1431 Ås, Norway; Gry.Alfredsen@nibio.no

3 Department of Wood Science and Technology, Biotechnical Faculty, University of Ljubljana, 1000 Ljubljana, Slovenia; Miha.Humar@bf.uni-lj.si (M.H.); Davor.Krzisnik@bf.uni-lj.si (D.K.); Bostjan.Lesar@bf.uni-lj.si (B.L.)

4 CATAS, 33048 San Giovanni al Natisone, Italy; conti@catas.com

5 LJ Cookson Consulting, Warrandyte, VIC 3113, Australia; laurie@ljcookson.com

6 Norwegian Institute of Wood Technology (NTI), 0314 Oslo, Norway; per.otto.flate@treteknisk.no (P.O.F.); ulrich.hundhausen@treteknisk.no (U.H.)

$7 \quad$ VTT Technical Research Centre of Finland, 02044 Espoo, Finland; stefania.fortino@vtt.fi

8 Department of Agriculture and Fisheries, Forestry Science, Ecosciences Precinct, Brisbane, QLD 4102, Australia; Lesley.Francis@daf.qld.gov.au (L.F.); jak.norton@gmail.com (J.N.)

9 Latvian State Institute of Wood Chemistry, 1006 Riga, Latvia; ilzeirbe@edi.lv

10 Institut für Holztechnologie Dresden (IHD), 01217 Dresden, Germany; Kordula.Jacobs@ihd-dresden.de

11 Danish Technological Institute (DTI), 2630 Taastrup, Denmark; mkl@teknologisk.dk

12 Thuenen Institute of Wood Research, 21031 Hamburg, Germany; eckhard.melcher@thuenen.de

13 Heinz-Piest-Institute of Craftsmen Techniques, 30167 Hannover, Germany; Meyer@hpi-hannover.de

14 National Centre for Timber Durability and Design Life (USC), University of the Sunshine Coast, Brisbane, QLD 4102, Australia; jmorrell@usc.edu.au

15 CNR IBE, Italian National Research Council, Institute of Bioeconomy, 50019 Sesto Fiorentino, Italy; sabrina.palanti@ibe.cnr.it

16 Department of Wood Science and Engineering, Oregon State University, Corvallis, OR 97331, USA; gerald.presley@oregonstate.edu

17 Faculty of Wood Sciences and Technology, Technical University in Zvolen, 96001 Zvolen, Slovakia; reinprecht@tuzvo.sk

18 SCION, Rotorua 3010, New Zealand; Tripti.Singh@scionresearch.com

19 FP Innovations, Vancouver, BC V6T 1Z4, Canada; Rod.Stirling@fpinnovations.ca

20 Natural Resources Institute Finland (LUKE), 57200 Savonlinna, Finland; martti.venalainen@luke.fi

21 Research Institute of Sweden (RISE), 50462 Borås, Sweden; mats.westin@ri.se

22 Faculty of Resource Science \& Technology, Universiti Malaysia Sarawak (Unimas), Kota Samarahan Sarawak 94300, Malaysia; ahhwong@unimas.my

23 Building Research Establishment, Garston, Watford WD25 9XX, UK; Ed.Suttie@bregroup.com

* Correspondence: christian.brischke@uni-goettingen.de

Abstract: Service life planning with timber requires reliable models for quantifying the effects of exposure-related parameters and the material-inherent resistance of wood against biotic agents. The Meyer-Veltrup model was the first attempt to account for inherent protective properties and the wetting ability of wood to quantify resistance of wood in a quantitative manner. Based on test data on brown, white, and soft rot as well as moisture dynamics, the decay rates of different untreated wood species were predicted relative to the reference species of Norway spruce (Picea abies). The present study aimed to validate and optimize the resistance model for a wider range of wood species including very durable species, thermally and chemically modified wood, and preservative treated wood. The general model structure was shown to also be suitable for highly durable materials, 
but previously defined maximum thresholds had to be adjusted (i.e., maximum values of factors accounting for wetting ability and inherent protective properties) to 18 instead of 5 compared to Norway spruce. As expected, both the enlarged span in durability and the use of numerous and partly very divergent data sources (i.e., test methods, test locations, and types of data presentation) led to a decrease in the predictive power of the model compared to the original. In addition to the need to enlarge the database quantity and improve its quality, in particular for treated wood, it might be advantageous to use separate models for untreated and treated wood as long as the effect of additional impact variables (e.g., treatment quality) can be accounted for. Nevertheless, the adapted Meyer-Veltrup model will serve as an instrument to quantify material resistance for a wide range of wood-based materials as an input for comprehensive service life prediction software.

Keywords: biological durability; dose-response model; fungal decay; moisture dynamics; moisture performance; service life prediction; water uptake and release; wetting ability

\section{Introduction}

Service life planning and performance prediction of wood are mutually linked. The first comprehensive approaches were in the Australian TimberLife project [1] and in different European research projects such as WoodExter [2], WoodBuild [3], and DuraTB [4]. The latter proposed engineering guidelines accounting for exposure- and resistance-related parameters that can be captured in terms of a dosage, which is a well-defined relationship with a response such as the biological depolymerization of wood. Among the numerous wood-destroying organisms, decay fungi play a vital role globally, since their spores are ubiquitous and can infest wood even under extreme climatic conditions [5,6].

The material-inherent resistance of wood against wood-destroying fungi is the product of chemical ingredients with inhibitory effects on fungal activity (e.g., extractives) and its repellency to moisture. Both can originate either from the natural chemical constitution and anatomy of the wood tissue itself or from man-made improvement through cell wall modification, water-repellant, and/or preservative treatments. The resulting mode of protective action is usually a combination of wood's inherent resistance and its wetting ability.

The durability of wood and wood-based materials against wood-destroying fungi is usually tested through incubation with monocultures of basidiomycetes under sterile conditions in the laboratory or exposure in field tests with or without ground contact. The moisture content and temperature of wood are kept at a favorable level for most decay fungi under standardized laboratory conditions. Permanent wetting of the wood samples is provided by high aqueous-containing nutritious media such as malt agar (e.g., EN 1132 [7]) or moist soil substrate in so-called soil-block tests (e.g., AWPA E10-16 [8]). Similarly, wood specimens that are buried to half of their length in soil-contact field tests (e.g., AWPA E7-15 [9] and EN 252 [10]) and in so-called semi-field tests performed with unsterile soil under laboratory conditions (terrestrial microcosms, e.g., ENV 807 [11]; CEN/TS 150832 [12], and AWPA E14-16 [13]) are permanently wet. Permanent wetting of test specimens may reduce the mode of protective action by leaching biocidal components. Moreover, this permanently wet condition poorly represents above-ground applications where the wood is exposed to cyclical wetting and drying. Under such conditions, the moisture performance of wood has a greater influence on service life. Wood materials that are low in biocidal components but exhibit high water-repellency, usually perform worse than expected compared with outdoor above-ground conditions where they can dry out and are consequently not constantly wet. Occasionally, this is called "torture testing" [14] since it does not necessarily reflect the anticipated use conditions. Above-ground field tests deliver durability data under quite realistic hazard conditions, but respective test data are sparsely available [15]. Numerous above-ground test methods have been reported, but very few are standardized [16]. Furthermore, they cover a wide range of above-ground use conditions representing different moisture-induced decay risks. In summary, the 
durability classification of wood rarely considers moisture performance. The European standard EN 350 [17] — at least in its current form —indicates that permeability to water should be considered. Permeability is defined as the "ease with which water penetrates a wood-based matrix (wood of a particular species, wood-based material) and is released by evaporation" [17]. Accordingly, the permeability to water and the rate of release can provide information relevant for wood's expected service life. However, guidance on the incorporation of this material property in the classification of biological durability of wood is still lacking, but essential for more accurately predicting the service life of timber structures.

Meyer-Veltrup et al. [18] approached predicting the service life of wood above-ground considering the combined effects of wetting ability and durability data. A comprehensive dataset was obtained from laboratory durability tests and still ongoing field tests in three different countries. In addition, four different wetting ability tests were performed with the same material. A dose-response concept was used to predict decay rates for specimens exposed above-ground using various indicating factors. The Meyer-Veltrup model was developed and optimized considering the resistance of wood to brown, white, and soft rot, as well as relevant types of water uptake and release.

Decay rates from above-ground field tests at different test sites in Norway were predicted with the model. In a second step, the model was validated using data from laboratory and field tests performed in Germany and Sweden. The model was next validated using data from laboratory and field tests by different authors [18-20]. Material resistance data were determined for additional wood species [21-23] and wood treatments [19,24].

The model was found to be fairly reliable and slightly conservative, and it had the advantage of being implemented into existing engineering design guidelines. Several reality checks revealed a sufficient model fit when linked with a climate exposure model and a decay prediction model [25], as reported for timber bridge structures [4]. However, Brischke et al. [26] reported that the general good fit of the Meyer-Veltrup model did not apply to preservative-treated wood or wood-based materials due to a lack of data on their inherent resistance and/or wetting ability. In addition, at the time, the optimized model did not include extremely durable materials. The most durable materials included in the original research were black locust, English oak, teak, and merbau [18]. The maximum value of modifying factors accounting for inherent protective properties $\left(k_{i n h}\right)$ and wetting ability of wood $\left(k_{w a}\right)$ were set to 5.0 as this gave the best fit with above-ground field test data. The maximum threshold might need to be higher for preservative-treated and modified wood. On the other hand, further factors may help account for treatment quality, preservative concentration and retention, modification level, superficial treatments, and coatings. However, it is difficult to quantify these factors, though attempts have been made to model such factors [27].

This study aimed to collect data on the inherent protective properties and the wetting ability of wood to further validate and optimize the Meyer-Veltrup model for material resistance. The range of wood-based materials included preservative-treated and modified woods tested under laboratory and above-ground field conditions in different parts of the world. To better utilize existing durability test data generated in different parts of the world but using different reference wood species, factors for different resistance parameters determined in Part 1 of this publication were applied in this study.

\section{Materials and Methods}

\subsection{The Meyer-Veltrup Model}

A model approach according to Meyer-Veltrup et al. [18] was applied to predict the above-ground field performance of the examined timbers. The model includes both the climatic exposure and the resistance of the material. Acceptance for a chosen design and material was expressed as: 
The exposure was expressed as an exposure dose $\left(D_{E d}\right)$ determined by daily averages of wood temperature $(T)$ and wood moisture content $(M C)$. The material property was expressed as a resistance dose $\left(D_{R d}\right)$. The dose would be expressed in days (d) with optimum moisture and temperature conditions for fungal decay as (see [28] and Equation (2)).

$$
D_{E d} \leq D_{R d}(\mathrm{~d})
$$

where:

$D_{E d}$ is the exposure dose (d);

$D_{R d}$ is the material resistance dose (d).

The exposure dose $D_{E d}$ depends on an annual dose at a specific geographical location and several factors describing the effect of driving rain, local climate, sheltering, distance from the ground, and design details. A detailed description of the development of the exposure module of the model is given by [3]. The present study focused on the resistance dose module of the model, which is the resistance, expressed as material resistance dose $D_{R d}$. The latter is the product of a critical dose $D_{\text {crit }}$ and two factors considering the wetting ability of wood $\left(k_{w a}\right)$ and its inherent durability $\left(k_{i n h}\right)$. The approach is described by Equation (3) according to [28]:

$$
D_{R d}=D_{c r i t} \times k_{w a} \times k_{i n h}(\mathrm{~d})
$$

where:

$D_{R d}$ is the material resistance dose (d);

$D_{\text {crit }}$ is the critical dose (d) corresponding to decay rating 1 (EN 252 [10]);

$k_{w a}$ is a factor accounting for the wetting ability of the material (-) relative to a reference wood species;

$k_{\text {inh }}$ is a factor accounting for the inherent protective properties of the material against decay (-) relative to a reference wood species.

The critical dose $\left(D_{\text {crit }}\right)$ was evaluated for Scots pine sapwood and Douglas fir heartwood according to [28]. The critical dose corresponding to decay rating " 1 " was more or less independent from the wood species. Instead, differences between species and/or treatments were better expressed by their moisture dynamics and decay inhibiting properties. For Scots pine sapwood and Douglas-fir heartwood, the critical dose was around 325 days with favorable conditions for fungal decay [28], as previously determined in horizontal double-layer experiments performed at several locations in Europe for up to eight years [29].

\subsection{Data Acquisition}

Data on material resistance based upon laboratory and field wood durability tests and different wetting ability tests were gathered from scientific publications, research reports, and technical guidelines. In addition, raw data in terms of mass loss, decay ratings, or moisture-related characteristics were provided by the authors. Information about the materials included in this study is summarized in Tables 1-4 as well as the respective data sources used to calculate the modifying factors $k_{w a}$ and $k_{i n h}$, and decay rates in in- and above-ground tests are indicated in Tables 5-8. Results from the in-ground field tests were used to supplement laboratory tests when accounting for soft rot resistance to calculate the respective factor $k_{i n h}$. 
Table 1. Untreated hardwoods included for validation and optimization of the material resistance model.

\begin{tabular}{|c|c|c|c|}
\hline Wood Species & Common Name & Origin & References \\
\hline Acer platanoides/A. pseudoplatanus & Norway maple/Sycamore & Europe & {$[18,30-34]$} \\
\hline Alnus glutinosa & Black alder & Europe & {$[18,31,34-36]$} \\
\hline Betula pendula/B. pubescens & Silver birch/Downy birch & Europe & {$[18,31,35,36]$} \\
\hline Dicorynia guianensis & Basralocus & S. America & {$[31,34,37-39]$} \\
\hline Fagus sylvatica & European beech & Europe & {$[15,18,19,31-36,39-49]$} \\
\hline Fraxinus excelsior & European ash & Europe & {$[18,19,31,35,36]$} \\
\hline Intsia bijuga & Merbau & Africa & {$[18,31,50,51]$} \\
\hline Lophira alata & Bongossi & Africa & {$[31,34,37-40,52,53]$} \\
\hline Peltogyne spp. & Amaranth & C. America & {$[30,34,38]$} \\
\hline Populus tremula & Aspen & Europe & {$[15,18,32,33,35,36]$} \\
\hline Quercus robur/Q. petraea & European oak & Europe & $\begin{array}{c}{[15,18,19,30,31,34-36,38-} \\
41,43,44,46,49,52-60]\end{array}$ \\
\hline Robinia pseudoacacia & Black locust & Europe & {$[18,31,34,36,39,49,52,53,57,61]$} \\
\hline Salix caprea & Goat willow & Europe & [18] \\
\hline Sorbus aucuparia & Rowan & Europe & {$[18,21]$} \\
\hline Tectona grandis & Teak & Asia & {$[18,31,34-36,38,39,62]$} \\
\hline Tilia cordata & Lime & Europe & [18] \\
\hline Ulmus glabra & Wych elm & Europe & {$[18,55]$} \\
\hline
\end{tabular}

Table 2. Untreated softwoods included for validation and optimization of the material resistance model. (sw $=$ sapwood).

\begin{tabular}{|c|c|c|c|}
\hline Wood Species & Common Name & Origin & References \\
\hline Abies alba & Silver fir & Europe & {$[18,57]$} \\
\hline Juniperus communis & Juniper & Europe & {$[18,21]$} \\
\hline Larix decidua & European larch & Europe & {$[18,19,31,35,36,39,42,45,48,49,55-57,63]$} \\
\hline Larix sibirica & Siberian larch & Europe & {$[15,18,31,41,42,60,64,65]$} \\
\hline Picea sitchensis & Sitka spruce & Europe & [18] \\
\hline Pinus spp. & Southern pine sw & N. America & {$[18,33,61,66]$} \\
\hline Pinus radiata & Radiata pine sw & New Zealand & {$[18,31,34]$} \\
\hline Pinus sylvestris & Scots pine sw & Europe & $\begin{array}{c}{[15,18,19,31,39,41-} \\
43,45,49,55,57,59,60,63,65,67,68] \\
{[18,19,31,33,34,39,41-} \\
43,45,48,49,56,57,61,63,68,69]\end{array}$ \\
\hline Pseudotsuga menziesii & $\begin{array}{l}\text { Douglas-fir } \\
\text { Douglas-fir sw }\end{array}$ & $\begin{array}{l}\text { N. America, Europe, } \\
\text { New Zealand }\end{array}$ & $\begin{array}{c}{[15,18,31,34,38-40,42,43,45,57,59,63,70-73]} \\
{[18,42,66,72]}\end{array}$ \\
\hline Thuja plicata & Western red-cedar & $\begin{array}{l}\text { N. America } \\
\text { Europe }\end{array}$ & $\begin{array}{c}{[15,18,30,31,39,58,63,71,73]} \\
{[18,55]}\end{array}$ \\
\hline
\end{tabular}

Table 3. Modified timbers included for validation and optimization of the material resistance model. (sw = sapwood, $\mathrm{HT}=$ heat treatment, $\mathrm{OHT}=$ oil-heat treatment, $\mathrm{AC}=$ acetylation, FA = furfurylation, DMDHEU = treatment with 1.3dimethylol-4.5-dihydroxyethyleneurea).

\begin{tabular}{|c|c|c|c|c|}
\hline Wood Species & Common Name & Origin & Modification & References \\
\hline Fagus sylvatica & European beech & Europe & HT & {$[19,48,74]$} \\
\hline Picea abies & Norway spruce & Europe & HT & {$[19,32,41,48,59,61,68,75]$} \\
\hline Pinus sylvestris & Scots pine & Europe & $\mathrm{HT}$ & {$[18,33,34,41,56,59-61,68,75,76]$} \\
\hline Fraxinus excelsior & European ash & Europe & OHT & [18] \\
\hline Picea abies & Norway spruce & Europe & $\mathrm{OHT}$ & {$[18,57]$} \\
\hline Pinus spp. & Southern pine sw & USA & $\mathrm{AC}$ & {$[18]$} \\
\hline Pinus sylvestris/P. radiata & $\begin{array}{c}\text { Scots pine } \\
\text { sw / Radiata pine sw }\end{array}$ & Europe/New Zealand & $\mathrm{AC}$ & {$[18,34,41,60,61,76,77]$} \\
\hline Acer platanoides & Norway maple & Europe & FA & {$[18,32,78]$} \\
\hline Pinus spp. & Southern pine sw & USÄ & FA & {$[18,32]$} \\
\hline Pinus sylvestris & Scots pine sw & Europe & FA & {$[18,60,79]$} \\
\hline Pinus sylvestris & Scots pine sw & Europe & DMDHEU & {$[18,24,34,76,80]$} \\
\hline
\end{tabular}


Table 4. Preservative-treated timbers considered for validation and optimization of the material resistance model. (sw = sapwood; $\mathrm{CCA}=$ chromated copper arsenate; $\mathrm{Cu}=$ copper; $\mathrm{EA}=$ ethanolamine; $\mathrm{OA}=$ octanoic acid; Quat = quaternary ammonium compounds).

\begin{tabular}{ccc}
\hline Wood Species & Preservative/Treatment & References \\
\hline & CCA 2 kg/m $/ \mathrm{m}^{3}$ & {$[18,61,65]$} \\
Pinus sylvestris sw & CCA $4 \mathrm{~kg} / \mathrm{m}^{3}$ & {$[18,32,33,41,76,79]$} \\
& CCA $9 \mathrm{~kg} / \mathrm{m}^{3}$ & {$[32,33,41,61,68,79]$} \\
\hline
\end{tabular}

Cu (II) sulphate low: $0.35 \%$ aqueous solution

$\mathrm{Cu}$ (II) sulphate high: $1.4 \%$ aqueous solution

CuEA low: $0.098 \% \mathrm{Cu} ; 0.51 \%$ EA

CuEA high: $0.39 \% \mathrm{Cu} ; 2.05 \%$ EA

Picea abies

CuEAOA low: $0.098 \% \mathrm{Cu} ; 0.51 \%$ EA; $0.07 \%$ OA

CuEAOA high: $0.39 \% \mathrm{Cu} ; 2.05 \%$ EA; $0.29 \%$ OA

[81]

CuEAOAQuat low: 0.098\% Cu; 0.51\% EA; 0.07\% OA; 0.098\% Quat

CuEAOAQuat high: $0.39 \% \mathrm{Cu} ; 2.05 \% \mathrm{EA} ; 0.29 \%$ OA; $0.39 \%$ Quat

BoronEAOAQuat low: 0.098\% B; 0.51\% EA; 0.07\% OA; 0.098\% Quat

BoronEAOAQuat high: 0.39\% B; 2.05\% EA; 0.29 OA; 0.39\% Quat

Picea abies,

Pinus sylvestris sw, Larix decidua
$\mathrm{Cu}$ (II) carbonate: $0.25 \%$, dipping $8-\mathrm{h}^{1}$ and $24-\mathrm{h}$

$\mathrm{Cu}$ (II) carbonate: $0.25 \%$, vacuum ${ }^{1}$

$\mathrm{Cu}$ (II) carbonate: $0.25 \%$, vacuum + pressure

$\mathrm{Cu}$ (II) carbonate: $0.5 \%$, dipping $8-\mathrm{h}^{1}$ and $24-\mathrm{h}$

$\mathrm{Cu}$ (II) carbonate: $0.5 \%$, vacuum

$\mathrm{Cu}$ (II) carbonate: $0.5 \%$, vacuum + pressure

\begin{tabular}{lll}
\hline Pinus sylvestris sw & Metal-free organic & [18,32]
\end{tabular}

${ }^{1}$ treatments were applied only to Scots pine sw and Norway spruce.

Table 5. Factors accounting for the protective inherent properties $\left(k_{i n h}\right)$ and moisture performance (wetting ability, $\left.k_{w a}\right)$, and relative decay rate in above-ground field tests of untreated hardwood species. (br = brown rot; wr = white rot; $L W U=$ liquid water uptake; $V U=$ vapor uptake; $W R=$ water release; $C W U=$ capillary water uptake; $v_{\text {rel. }}=$ relative decay rate).

\begin{tabular}{|c|c|c|c|c|c|c|c|c|}
\hline \multirow{2}{*}{ Wood Species } & \multicolumn{3}{|c|}{$k_{i n h}$} & \multicolumn{4}{|c|}{$k_{w a}$} & \multirow{2}{*}{$v_{\text {rel. }}$. } \\
\hline & br & wr & Soil & $L W U$ & $V U$ & $W R$ & CWU & \\
\hline Acer platanoides/A. pseudoplatanus & 2.54 & 0.94 & 1.02 & 0.80 & 1.14 & 1.07 & 1.02 & 0.90 \\
\hline Alnus glutinosa & 1.09 & 1.04 & 0.72 & 0.87 & 1.14 & 1.16 & 1.06 & 1.35 \\
\hline Betula pendula/B. pubescens & 1.01 & 0.93 & 0.88 & 0.76 & 1.16 & 1.33 & 0.36 & 0.95 \\
\hline Dicorynia guianensis & 15.30 & 16.54 & 5.11 & 1.43 & 1.35 & 1.25 & 1.06 & 0.19 \\
\hline Fagus sylvatica & 1.26 & 0.70 & 0.61 & 0.73 & 1.15 & 1.30 & 1.44 & 1.17 \\
\hline Fraxinus excelsior & 6.62 & 0.75 & 1.30 & 1.01 & 1.17 & 1.07 & 0.74 & 0.39 \\
\hline Intsia bijuga & 16.19 & 9.93 & 16.33 & 1.72 & 1.63 & 0.55 & 4.62 & 0.25 \\
\hline Lophira alata & 13.26 & 14.64 & 10.52 & 1.54 & 1.58 & 1.28 & 1.24 & 0.19 \\
\hline Peltogyne spp. & 16.48 & 18.00 & 5.11 & 2.41 & 1.31 & 2.17 & 1.39 & 0.25 \\
\hline Populus tremula & 1.24 & 0.99 & 0.94 & 0.91 & 1.13 & 1.09 & 0.65 & 1.04 \\
\hline Quercus robur/Q. petraea & 14.62 & 8.04 & 2.77 & 1.70 & 1.34 & 1.70 & 0.88 & 0.47 \\
\hline Robinia pseudoacacia & 12.20 & 12.34 & 2.67 & 2.67 & 2.15 & 1.24 & 1.65 & 0.24 \\
\hline Salix caprea & 1.37 & 1.16 & 1.46 & 1.16 & 1.36 & 0.85 & 0.60 & 0.50 \\
\hline Sorbus aucuparia & 1.27 & 1.25 & 1.46 & 0.94 & 1.00 & 1.07 & 0.44 & 0.56 \\
\hline Tectona grandis & 18.00 & 16.96 & 7.83 & 2.08 & 2.43 & 1.04 & 1.17 & 0.16 \\
\hline Tilia cordata & 0.89 & 1.04 & 1.39 & 0.92 & 1.43 & 0.83 & 0.38 & 0.86 \\
\hline Ulmus glabra & 7.33 & 1.12 & 1.66 & 1.14 & 1.31 & 0.91 & 0.49 & 0.39 \\
\hline
\end{tabular}


Table 6. Factors accounting for the protective inherent properties $\left(k_{i n h}\right)$ and moisture performance (wetting ability, $\left.k_{w a}\right)$, and relative decay rate in above-ground field tests of untreated softwood species. (br = brown rot; $w r=$ white rot; $L W U=$ liquid water uptake; $V U=$ vapor uptake; $W R=$ water release; $C W U=$ capillary water uptake; $v_{\text {rel. }}=$ relative decay rate; $\mathrm{sw}=$ sapwood).

\begin{tabular}{|c|c|c|c|c|c|c|c|c|}
\hline \multirow{2}{*}{ Wood Species } & \multicolumn{3}{|c|}{$k_{i n h}$} & \multicolumn{4}{|c|}{$k_{w a}$} & \multirow{2}{*}{$v_{\text {rel. }}$. } \\
\hline & br & wr & soil & $L W U$ & $V U$ & $W R$ & CWU & \\
\hline Abies alba & 1.33 & 1.22 & 1.24 & 0.91 & 1.09 & 0.96 & 0.68 & 1.14 \\
\hline Juniperus communis & 13.05 & 13.11 & 7.53 & 1.30 & 1.43 & 0.77 & 1.20 & 0.32 \\
\hline Larix decidua & 4.13 & 6.15 & 2.30 & 1.81 & 1.39 & 0.98 & 1.87 & 0.34 \\
\hline Larix sibirica & 3.32 & 1.55 & 4.86 & 1.01 & 1.30 & 1.05 & 0.46 & 0.45 \\
\hline Picea sitchensis & 1.09 & 1.82 & 1.14 & 1.17 & 1.03 & 1.03 & 3.92 & 0.86 \\
\hline Pinus spp. sw (Southern pine) & 2.38 & 10.88 & 0.87 & 0.89 & 1.01 & 0.69 & 0.58 & 0.76 \\
\hline Pinus radiata sw & 1.95 & 0.89 & 1.16 & 0.63 & 0.92 & 1.29 & 0.85 & 0.98 \\
\hline Pinus sylvestris & 2.98 & 6.85 & 1.86 & 1.06 & 1.21 & 0.89 & 1.37 & 0.47 \\
\hline P. sylvestris sw & 0.97 & 0.91 & 1.14 & 1.00 & 1.08 & 0.88 & 1.03 & 0.83 \\
\hline Pseudotsuga menziesii & 3.46 & 9.30 & 3.34 & 1.61 & 1.25 & 0.93 & 2.84 & 0.55 \\
\hline P. menziesii sw & 1.63 & 4.66 & 1.43 & 1.14 & 1.11 & 0.92 & 1.01 & 0.83 \\
\hline Thuja plicata (N.-America) & 16.73 & 11.67 & 2.63 & 1.13 & 1.61 & 0.59 & 0.27 & 0.42 \\
\hline T. plicata (Europe) & 18.00 & 11.10 & 1.31 & 0.78 & 1.29 & 0.81 & 0.56 & 0.35 \\
\hline
\end{tabular}

Table 7. Factors accounting for the protective inherent properties $\left(k_{i n h}\right)$ and moisture performance (wetting ability, $\left.k_{w a}\right)$, and relative decay rate in above-ground field tests of modified timbers. (br = brown rot; $w r=$ white rot; $L W U=$ liquid water uptake; $V U=$ vapor uptake; $W R=$ water release; $C W U=$ capillary water uptake; $v_{\text {rel. }}=$ relative decay rate; $\mathrm{TM}$ = thermal modification; OHT = oil-heat treatment; $\mathrm{AC}=$ acetylation; FA = furfurylation; DMDHEU = treatment with 1.3-dimethylol-4.5-dihydroxyethyleneurea).

\begin{tabular}{|c|c|c|c|c|c|c|c|c|}
\hline \multirow{2}{*}{ Wood Species and Treatment } & \multicolumn{3}{|c|}{$k_{i n h}$} & \multicolumn{4}{|c|}{$k_{w a}$} & \multirow{2}{*}{$v_{\text {rel. }}$} \\
\hline & br & $\mathbf{w r}$ & Soil & $L W U$ & $V U$ & $W R$ & CWU & \\
\hline F. sylvatica-TM & 9.60 & 7.60 & 4.68 & 1.79 & 2.86 & 0.43 & 3.24 & 0.02 \\
\hline P. abies-TM & 8.67 & 4.95 & 2.98 & 6.99 & 2.09 & 6.44 & 1.40 & 0.34 \\
\hline P. sylvestris-TM & 8.74 & 9.72 & 5.36 & 1.87 & 1.80 & 1.70 & 1.47 & 0.53 \\
\hline F. excelsior-OHT & 18.00 & 9.71 & 11.79 & 1.99 & 2.91 & 0.60 & 1.60 & 0.07 \\
\hline P. abies-OHT & 18.00 & 18.00 & 9.66 & 1.73 & 2.35 & 0.51 & 0.88 & 0.16 \\
\hline Pinus spp. sw (Southern pine)-AC & 18.00 & 18.00 & 17.78 & 1.32 & 2.86 & 0.31 & 0.76 & 0.04 \\
\hline P. sylvestris $P$. radiata $\mathrm{sw}-\mathrm{AC}$ & 18.00 & 18.00 & 16.69 & 1.57 & 3.01 & 1.43 & 1.28 & 0.07 \\
\hline A. platanoides-FA & 14.72 & 10.09 & 3.86 & 1.89 & 2.92 & 0.23 & 1.08 & 0.05 \\
\hline Pinus spp. sw (Southern pine)-FA & 10.88 & 12.67 & 6.54 & 1.73 & 2.23 & 0.40 & 1.45 & 0.12 \\
\hline P. sylvestris sw-FA & 18.00 & 18.00 & 7.53 & 2.79 & 3.30 & 0.23 & 1.54 & 0.27 \\
\hline
\end{tabular}

Table 8. Factors accounting for the protective inherent properties $\left(k_{i n h}\right)$ and moisture performance (wetting ability, $\left.k_{w a}\right)$, and relative decay rate in above-ground field tests of preservative-treated timbers. ( $\mathrm{br}=$ brown rot; wr $=\mathrm{white}$ rot; $L W U=$ liquid water uptake; $V U=$ vapor uptake; $W R=$ water release; $C W U=$ capillary water uptake; $v_{\text {rel }}=$ relative decay rate; $\mathrm{CCA}$ = chromated copper arsenate; $\mathrm{Cu}=$ copper; $\mathrm{EA}=$ ethanolamine; $\mathrm{OA}=$ octanoic acid; Quat = quaternary ammonium compounds; P.s. = Pinus sylvestris; P.a. = Picea abies; L.d. = Larix decidua).

\begin{tabular}{|c|c|c|c|c|c|c|c|c|}
\hline \multirow{2}{*}{ Wood Species and Treatment } & \multicolumn{3}{|c|}{$k_{i n h}$} & \multicolumn{4}{|c|}{$k_{w a}$} & \multirow{2}{*}{$v_{\text {rel. }}$. } \\
\hline & br & wr & Soil & $L W U$ & $V U$ & $W R$ & $C W U$ & \\
\hline P. sylvestris, CCA, $2 \mathrm{~kg} / \mathrm{m}^{3}$ & 18.00 & 18.00 & 5.12 & 0.92 & 0.97 & 0.87 & 2.49 & 0.10 \\
\hline P. sylvestris, CCA, $4 \mathrm{~kg} / \mathrm{m}^{3}$ & 18.00 & 18.00 & 7.79 & 1.34 & 0.92 & 1.22 & 1.35 & 0.13 \\
\hline P. sylvestris, CCA, $9 \mathrm{~kg} / \mathrm{m}^{3}$ & 9.66 & 18.00 & 11.87 & 0.83 & 1.02 & 0.88 & 1.02 & 0.06 \\
\hline P. abies, Cu (II) sulph. low & 6.75 & 10.37 & 1.82 & 0.90 & 0.80 & 0.97 & 1.04 & 0.69 \\
\hline P. abies, Cu (II) sulph. high & 8.77 & 10.54 & 2.66 & 0.89 & 0.77 & 1.31 & 0.81 & 0.63 \\
\hline P. abies, CuEA low & 7.13 & 8.94 & 2.37 & 0.88 & 0.90 & 1.28 & 0.94 & 0.61 \\
\hline P. abies, CuEA high & 8.00 & 7.15 & 2.00 & 0.97 & 0.92 & 0.90 & 1.10 & 0.65 \\
\hline P. abies, CuEAOA low & 6.48 & 8.80 & 1.72 & 1.04 & 0.98 & 1.16 & 0.91 & 0.11 \\
\hline P. abies, CuEAOA high & 7.34 & 6.12 & 1.98 & 0.98 & 0.93 & 1.06 & 1.50 & 0.57 \\
\hline P. abies, CuEAOAQuat low & 13.87 & 9.95 & 1.45 & 0.87 & 1.03 & 0.94 & 0.83 & 0.21 \\
\hline P. abies, CuEAOAQuat high & 16.42 & 7.78 & 1.84 & 0.90 & 1.10 & 0.75 & 1.16 & 0.01 \\
\hline P. abies, BorEAOAQ̄uat low & 12.53 & 9.76 & 0.85 & 0.99 & 0.89 & 0.95 & 1.41 & 0.86 \\
\hline P. abies, BorEAOAQuat high & 13.00 & 8.30 & 0.88 & 1.08 & 0.75 & 1.08 & 4.28 & 0.61 \\
\hline
\end{tabular}


Table 8. Cont.

\begin{tabular}{|c|c|c|c|c|c|c|c|c|}
\hline \multirow{2}{*}{ Wood Species and Treatment } & \multicolumn{3}{|c|}{$k_{\text {inh }}$} & \multicolumn{4}{|c|}{$k_{w a}$} & \multirow{2}{*}{$v_{\text {rel. }}$} \\
\hline & br & wr & Soil & LWU & $V U$ & $W R$ & $C W U$ & \\
\hline P. abies, Cu 0.25\%, dip. 8-h & 11.43 & 16.02 & 1.47 & 1.19 & 0.83 & 0.51 & 0.79 & 0.58 \\
\hline P. abies, Cu $0.25 \%$, dip. $24-\mathrm{h}$ & 13.71 & 18.00 & 1.71 & 1.17 & 0.74 & 0.78 & 0.71 & 0.46 \\
\hline P. abies, $\mathrm{Cu} 0.25 \%$, vac. & 18.00 & 18.00 & 3.57 & 1.14 & 0.73 & 0.77 & 0.81 & 0.17 \\
\hline P. abies, Cu $0.25 \%$, vac. + press. & 16.01 & 15.30 & 4.50 & 1.20 & 0.83 & 0.45 & 0.75 & 0.03 \\
\hline P. abies, Cu $0.5 \%$, dip. 8-h & 13.76 & 18.00 & 1.54 & 1.21 & 0.81 & 0.36 & 1.01 & 0.39 \\
\hline P. abies, $\mathrm{Cu} 0.5 \%$, dip. $24-\mathrm{h}$ & 14.48 & 18.00 & 2.94 & 1.16 & 0.83 & 0.33 & 1.02 & 0.42 \\
\hline P. abies, $\mathrm{Cu} 0.5 \%$, vac. & 15.35 & 15.25 & 3.18 & 1.25 & 0.80 & 0.32 & 0.97 & 0.13 \\
\hline P. abies, Cu $0.5 \%$, vac. + press. & 15.19 & 15.07 & 3.60 & 1.29 & 0.72 & 0.45 & 0.88 & 0.15 \\
\hline P. sylvestris, $\mathrm{Cu} 0.25 \%$, dip. $8-\mathrm{h}$ & 13.27 & 10.20 & 1.39 & 1.31 & 0.90 & 0.36 & 4.96 & 0.16 \\
\hline P. sylvestris, Cu $0.25 \%$, dip. $24-\mathrm{h}$ & 13.69 & 11.08 & 2.38 & 1.34 & 0.88 & 0.30 & 1.86 & 0.09 \\
\hline P. sylvestris, Cu $0.25 \%$, vac. & 18.00 & 18.00 & 2.01 & 1.28 & 0.80 & 0.53 & 2.64 & 0.09 \\
\hline P. sylvestris, Cu $0.25 \%$, vac. + press. & 18.00 & 17.64 & 3.03 & 1.14 & 0.76 & 0.33 & 1.81 & 0.00 \\
\hline P. sylvestris, Cu 0.5\%, dip. 8-h & 13.83 & 14.45 & 2.55 & 1.20 & 0.77 & 0.26 & 2.63 & 0.13 \\
\hline P. sylvestris, Cu 0.5\%, dip. 24-h & 16.94 & 15.84 & 2.75 & 1.21 & 0.76 & 0.32 & 2.23 & 0.09 \\
\hline P. sylvestris, Cu $0.5 \%$, vac. & 17.23 & 18.00 & 3.59 & 1.22 & 0.67 & 0.53 & 1.60 & 0.03 \\
\hline P. sylvestris, $\mathrm{Cu} 0.5 \%$, vac. + press. & 15.49 & 17.34 & 3.28 & 1.22 & 0.65 & 0.37 & 2.73 & 0.00 \\
\hline L. decidua, Cu $0.25 \%$, dip. 24-h & 11.94 & 11.58 & 1.03 & 2.10 & 0.85 & 0.54 & 15.49 & 0.00 \\
\hline L. decidua, Cu $0.25 \%$, vac. + press. & 17.99 & 18.00 & 1.10 & 1.85 & 0.84 & 0.28 & 5.63 & 0.17 \\
\hline L. decidua, Cu 0.5\%, dip. 24-h & 13.84 & 14.53 & 1.14 & 2.02 & 0.94 & 0.18 & 4.30 & 0.09 \\
\hline L. decidua, $\mathrm{Cu} 0.5 \%$, vac. & 17.60 & 18.00 & 0.87 & 2.03 & 0.93 & 0.28 & 17.00 & 0.06 \\
\hline L. decidua, Cu $0.5 \%$, vac. + press. & 14.78 & 14.00 & 1.32 & 1.73 & 0.76 & 0.19 & 4.42 & 0.20 \\
\hline P. sylvestris, metal-free organic & 18.00 & 18.00 & 2.41 & 0.85 & 1.05 & 0.78 & 0.48 & 0.09 \\
\hline
\end{tabular}

\subsection{Test Methods for Determining the Modifying Factors $k_{i n h}$ and $k_{w a}$}

Meyer-Veltrup et al. [18] determined the modifying factors $k_{i n h}$ and $k_{w a}$ on the basis of different laboratory durability test methods against brown, white, and soft rot-causing fungi and different moisture performance tests accounting for liquid water uptake during submersion, water vapor uptake at high relative humidity $(R H)$, desorption tests at low $R H$ (approx. 0\%), and capillary water uptake $(C W U)$ of end-grain surfaces. The test protocols are described in detail in Part 1 of this publication [83]. In each case, the reference wood species was Norway spruce (Picea abies). This study enlarged the pool of datasets and also included results where Scots pine sapwood (Pinus sylvestris) and European beech (Fagus sylvatica) were used as reference species. Factors accounting for the relationship between the material resistance and its respective components for the three reference species were applied as described in Part 1 [83] of this publication. In addition to standard basidiomycete tests with brown and white rot fungi (e.g., EN 113-2 [7]) and soil contact soft rot tests under laboratory (e.g., ENV 807 [11]) and field conditions (e.g., EN 252 [10]), results from basidiomycete mini-block tests [84] were considered. Results from submersion and floating tests according to CEN/TS 16818 [85] were considered for calculating $k_{w a}$ factors in addition to the tests described in Part 1 [83].

Furthermore, results of above-ground tests performed at different locations worldwide were obtained in horizontal lap-joint tests $[45,69,86]$, sandwich tests [16], decking tests [19], deck tests [63,81], close-to-ground mini-stake tests [79], multiple layer tests [15], block tests [79-81], vertically hanging stakes [57], painted and unpainted L-joint tests [15,87], horizontal double layer tests [57], and modified horizontal double layer tests [68].

A decay rating of specimens in and above-ground was performed regularly (usually once per year) with the help of a pick test. The depth and distribution of decay were determined and rated using the five-step scheme according to EN 252 [10] as follows: 0 = sound, 1 = slight attack, 2 = moderate attack, 3 = severe attack, and $4=$ failure. Some studies used the American and/or Australian rating system (10 to 0), which were transformed to the EN 252 scale as suggested by Stirling et al. [88]. 
The normal process requires that all specimens of a specific material must have reached decay rating 4 to determine mean lifetime. This was not the case for all materials. Therefore, the mean decay rate $v_{\text {mean }}$ was calculated according to [18] as follows (Equation (4)):

$$
v_{\text {mean }}=\frac{\sum_{i}^{n} v_{i}}{n}=\frac{\sum_{i}^{n} \frac{R}{t}}{n}
$$

where:

$v_{\text {mean }}$ is the mean decay rate of specimens $\left(\mathrm{a}^{-1}\right)$;

$v_{i}$ is the decay rate of single specimens $\left(\mathrm{a}^{-1}\right)$;

$R$ is the decay rating according to EN 252 [10];

$t$ is the exposure time (a);

$n$ is the number of replicate specimens (-).

For further modeling, a relative decay rate was calculated with Norway spruce as a reference species (Equation (5)) to become independent of the respective test location and test method. When other wood species than Norway spruce were used as reference, species- or genus-specific factors were established for correcting the values according to the relationships between reference species and Norway spruce.

$$
v_{\text {rel. }}=\frac{v_{\text {species } x}}{v_{\text {reference }}}
$$

where:

$v_{\text {rel. }}$ is the relative decay rate (-);

$v_{\text {species } x}$ is the decay rate of species $x\left(\mathrm{a}^{-1}\right)$;

$v_{\text {reference }}$ is the decay rate of a reference, here: Norway spruce, $\left(a^{-1}\right)$.

\subsection{Evaluation Procedure and Model Fitting}

Negative mass losses $(M L)$, i.e., mass gains, in laboratory decay tests were considered to be equal to zero for further calculations. To avoid unrealistically high relative values (factors), a threshold (Thr) was set to 18.0 for both factors, leaving the values in the following range: $0<k_{w a} \leq T h r$ and $0<k_{i n h} \leq T h r$ according to the best fit of the model based on the method of least squares.

The above-ground performance of wood was the target measure in this study. Performance was quantified by calculating the resistance dose $\left(D_{R d}\right)$ according to Equation (3) above, averaged for all available datasets per material (Tables 5-8), and used for optimizing the resistance model using a power regression function and the method of least squares.

To identify the most suitable indicators, different factors and factor combinations (based on results from the wetting ability and durability tests) were used to correlate the relative resistance dose $D_{R d, \text { rel. }}$ (Equation (6)) with the relative mean decay rate $\left(v_{\text {rell }}\right)$. Therefore, both measures were set relative to the reference species Norway spruce. To calculate $k_{\text {inh }}$ from laboratory basidiomycete tests (Equation (7)), $M L$ was factorized and either used as mean of $M L$ caused by brown and white rot (br:wr,mean) or as worst case, i.e., the maximum relative $M L$ representing white, brown, and soft rot, and exposure to soil, and thus the minimum $k_{\text {inh }}(\mathrm{min})$. Factors obtained in laboratory soil bed tests and in-ground field tests were averaged or $k_{i n h}$ was calculated from soft rot tests with soil contact $\left(k_{\text {inh,soil }}\right)$. 


$$
D_{R d, \quad \text { rel. }}=\frac{D_{R d, \text { species } x}}{D_{R d, \text { reference }}}
$$

where:

$D_{R d, r e l .}$ is the relative resistance dose (-);

$D_{R d \text {,species } x}$ is the resistance dose of species $\mathrm{x}\left(\mathrm{a}^{-1}\right)$;

$D_{R d}$ is the resistance dose of the reference, here: Norway spruce, $\left(\mathrm{a}^{-1}\right)$.

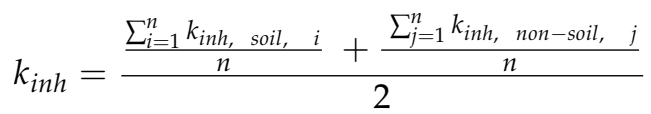

where:

$k_{i n h}$ is the factor accounting for the inherent protective properties of the material against decay (-);

$k_{\text {inh,soil, } i}$ is the factor accounting for the inherent protective properties of the material against decay in tests with soil contact (-);

$k_{i n h, n o n-s o i l, j}$ is the factor accounting for the inherent protective properties of the material against decay in tests without soil contact (-);

$n$ is the number of tests.

\section{Results and Discussion}

\subsection{Untreated Timber}

The results from the different moisture performance and durability tests are given in Tables 5-8 and are expressed as factors $k_{w a}$ and $k_{i n h}$. The results differed markedly between wood species, treatments, and test methods. The factor accounting for protective inherent properties $k_{\text {inh }}$ varied among the hardwoods between 0.61 (beech, soil) and 18.0 (Amaranth, white rot, and teak, brown rot, Table 5), i.e., the Thr for both factors, $k_{i n h}$ and $k_{w a}$ (see Section 2.4. Evaluation Procedure and Model fitting). The factors accounting for moisture performance (wetting ability, $k_{w a}$ ) showed less variation, i.e., between 0.38 (lime, $C W U$ ) and 2.43 (teak, liquid water uptake $(L W U)$ ). Similarly, $k_{i n h}$ varied more than $k_{w a}$ among the softwood species (Table 6).

The relative decay rate $\left(v_{\text {rel. }}\right)$ was between 0.16 (teak) and 1.35 (alder) among the hardwoods and between 0.32 (juniper) and 1.14 (silver fir) among the softwoods. The wider range in biological durability of hardwood species compared to softwoods is consistent with previous reports $[15,89]$.

\subsection{Modified Timber}

In general, both factors, $k_{i n h}$ and $k_{w a}$, of differently modified timber were higher compared to respective untreated wood species and showed lower $v_{\text {rel }}$. in above-ground durability tests (Table 7). The factor $k_{w a}$ varied between 0.43 and 3.24 for heat-treated beech, which also showed the lowest $v_{r e l}$. In contrast, thermally modified Norway spruce and Scots pine also showed rather high $k_{\text {inh }}$ (2.98-9.39), but suffered from comparatively high $v_{\text {rel., }}$ i.e., 0.34 and 0.53 , respectively. The latter might at least partly be explained through the increased brittleness of thermally modified wood [89], which can be mistaken for fungal decay, since the effect of high temperatures and brown rot decay on wood's structural integrity is very similar $[90,91]$. Thus, the severity of fungal decay in thermally modified wood can easily be overestimated.

\subsection{Preservative-Treated Timber}

The preservative-treated timbers generally showed high $k_{\text {inh }}$ values, often close to or at $T h r=18.0$. In contrast, the wetting ability factors were at least partly negatively affected by the treatments. In particular, many copper-treated woods showed low $k_{w a}$ values in desorption tests, i.e., 0.18 at minimum, which can be explained by increased sorption of wood after impregnation with aqueous salt solutions [92] and by the presence of quaternary 
ammonium compounds. These chemicals are known as surfactants and can therefore lower the surface tension between treated wood and water [93].

The summary table suggests that $k_{i n h}$ is dependent on the wood species (i.e., initial durability, permeability), the type and concentration of the wood preservative, and the impregnation process (Table 8). Furthermore, one needs to consider the type of decay and respective test fungi, such as copper-tolerant brown rot fungi, which make co-biocides play an important role in copper-containing preservatives [94]. This became particularly evident for wood treated with preservatives containing only copper as the active ingredient. In addition, the unexpectedly high $v_{\text {rel. }}$ might be attributed to the leaching of active ingredients such as boron, which led to high $k_{\text {inh }}$ values in laboratory tests without pre-aging.

Copper-based treatments are generally effective in the laboratory against fungal monocultures. Even shell treatments can be sufficient to prevent hyphal penetration to the central part of the specimens. However, surface cracks are formed when larger specimens are exposed outdoors, which enables access to the specimen core by decay fungi. In addition, leaching of active ingredients contributes to the higher susceptibility of wood to decay as well. However, copper mobility has also been shown to protect checks that expose untreated wood from the germination of basidiospores from copper-tolerant fungi $[95,96]$. Copper-ethanolamine-treated wood was considerably less effective against soft rot fungi than CCA-treated wood. On the one hand, this is likely the result of increased copper leaching during exposure to terrestrial microcosm. Up to $80 \%$ of the copper from the specimens is leached during such tests due to humic acids in compost soil and other acids excreted by fungi and bacteria [97]. On the other hand, arsenic also serves as an efficient co-biocide against soft rot fungi.

\subsection{Model Fitting}

The most suitable factors and factor combinations, respectively based on results from the wetting ability and durability tests, can be extracted from Table 9 where the coefficients of determination $\mathrm{R}^{2}$ for the various combinations are summarized. One of the best fits $\left(\mathrm{R}^{2}=0.336\right.$; Figure $\left.1 \mathrm{c}\right)$ between $v_{\text {rel. }}$ (Equation (5)) and the relative resistance dose $\left(D_{R d, \text { rel }}\right)$ (Equation (6)) was achieved by using the mean value of the four $k_{w a}$ factors multiplied with the $k_{i n h}$ factors based on soil contact tests and $k_{i n h}$ factors based on non-soil contact tests weighted equally (Equation (7)). This pair of factors led to the best fit already in the initial material resistance model presented by Meyer-Veltrup et al. [18]. However, the fit itself was poor and a slightly better fit $\left(\mathrm{R}^{2}\right.$ up to 0.376$)$ was reached when $k_{w a}$ was calculated based on CWU data and combined with $k_{i n h}$, either based on mean, minimum, or equally weighted values from soil and non-soil tests. However, the latter was mostly attributed to a series of tests performed with softwood species treated with different copper systems (Table 8); these showed highly varying CWU and thus contributed to higher prominence of a relationship between the relative $D_{R d}$ and $v_{r e l}$., which as such was only poorly fitted (see Figure 1b). 

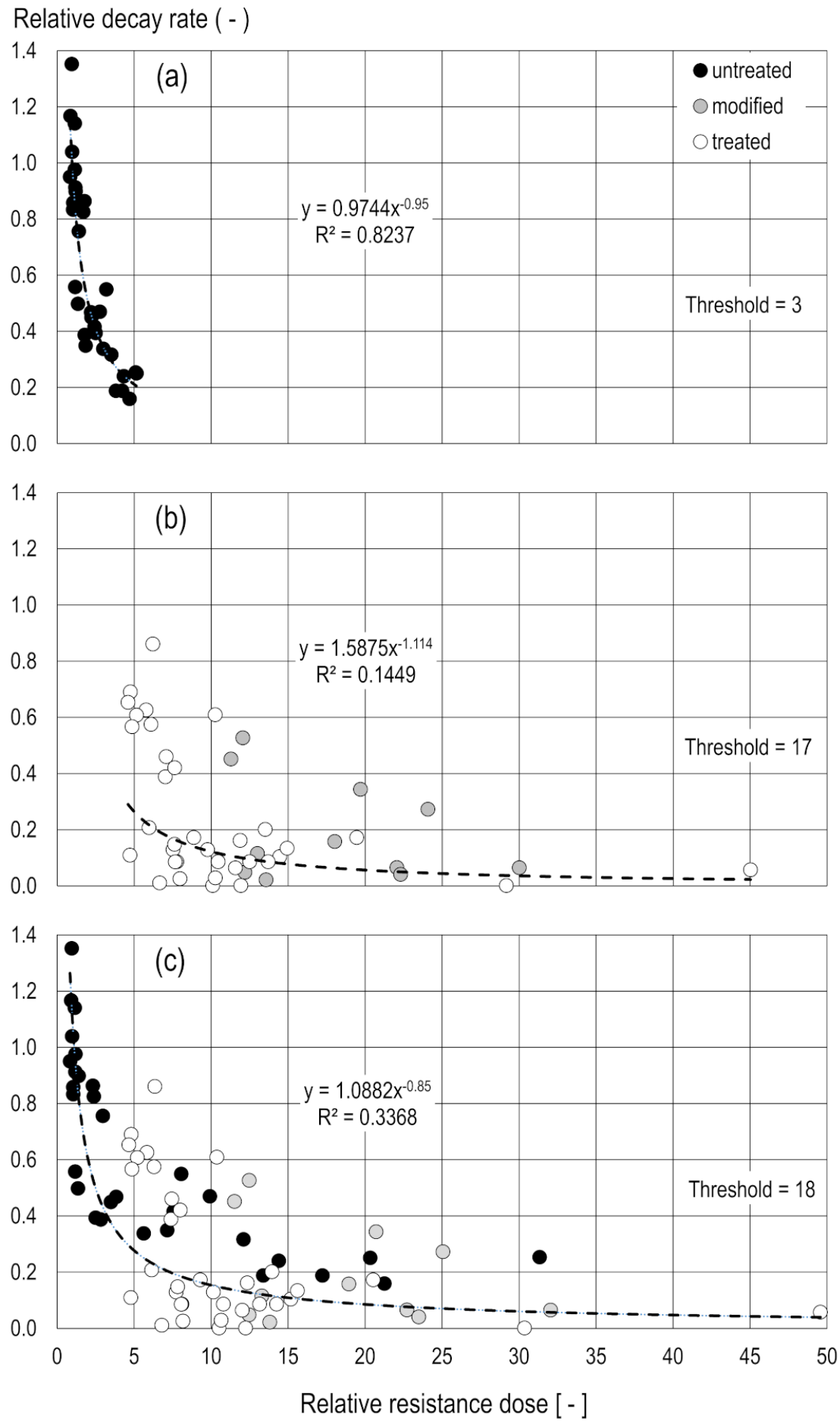

Figure 1. Relationship between calculated relative $D_{R d}$ and relative mean decay rate of specimens exposed in various above-ground field tests. The basis was 30 untreated timbers (a), 45 modified and preservative treated timbers (b), and both groups together (c). The $k_{i n h}$ factors based on soil contact tests and those based on non-soil contact tests (i.e., monocultures of brown and white rot fungi) were weighted equally; $k_{w a}$ factors of all wetting ability tests were weighted equally. 
Table 9. Coefficient of determination $\mathrm{R}^{2}$ for the relationship between the relative resistance dose $D_{R d}$ and the relative mean decay rate $\left(v_{\text {rell }}\right)$ in above-ground durability tests. $D_{R d}$ was calculated based on different combinations of factors accounting for protective inherent properties $\left(k_{i n h}\right)$ and moisture performance (wetting ability, $k_{w a}$ ) using data from the references provided in Tables 5-8. First line: $\mathrm{R}^{2}$ at a threshold of 17 , second line $\mathrm{R}^{2}$ with the threshold in brackets that gave the best fit. $(\mathrm{br}=$ brown rot; $\mathrm{wr}=$ white $\mathrm{rot} ; L W U=$ liquid water uptake; $V U=$ vapor uptake; $W R=$ water release; $C W U=$ capillary water uptake).

\begin{tabular}{|c|c|c|c|c|c|c|c|}
\hline \multirow{2}{*}{$k_{i n h}$} & \multicolumn{6}{|c|}{$k_{w a}$} & \multirow[b]{2}{*}{$1^{3}$} \\
\hline & $L W U$ & $V U$ & $W R$ & CWU & Mean ${ }^{1}$ & $\operatorname{Min}^{2}$ & \\
\hline Soil & $\begin{array}{c}0.133 \\
0.218(2)\end{array}$ & $\begin{array}{c}0.059 \\
0.072(4)\end{array}$ & $\begin{array}{c}0.000 \\
0.202(1)\end{array}$ & $\begin{array}{c}0.291 \\
0.295(14)\end{array}$ & $\begin{array}{c}0.168 \\
0.202(3)\end{array}$ & $\begin{array}{c}0.010 \\
>0.015(\infty)\end{array}$ & $\begin{array}{c}0.110 \\
0.166(3)\end{array}$ \\
\hline br:wr,Mean & $\begin{array}{c}0.298 \\
0.299(26)\end{array}$ & $\begin{array}{c}0.255 \\
0.267(34)\end{array}$ & $\begin{array}{c}0.076 \\
0.112(38)\end{array}$ & $\begin{array}{c}0.369 \\
0.375(36)\end{array}$ & $\begin{array}{c}0.341 \\
-\end{array}$ & $\begin{array}{c}0.138 \\
0.172(36)\end{array}$ & $\begin{array}{c}0.311 \\
0.313(26)\end{array}$ \\
\hline $\operatorname{Min}^{2}$ & $\begin{array}{c}0.150 \\
0.241(2)\end{array}$ & $\begin{array}{c}0.073 \\
0.087(4)\end{array}$ & $\begin{array}{c}0.000 \\
>0.002(\infty)\end{array}$ & $\begin{array}{c}0.376 \\
0.381(36)\end{array}$ & $\begin{array}{c}0.189 \\
0.217(3)\end{array}$ & $\begin{array}{c}0.018 \\
>0.021(\infty)\end{array}$ & $\begin{array}{c}0.133 \\
0.190(3)\end{array}$ \\
\hline Soil:no Soil,Mean & $\begin{array}{c}0.288 \\
0.291(27)\end{array}$ & $\begin{array}{c}0.220 \\
0.241(35)\end{array}$ & $\begin{array}{c}0.045 \\
0.081(38)\end{array}$ & $\begin{array}{c}0.377 \\
0.383(36)\end{array}$ & $\begin{array}{c}0.337 \\
-\end{array}$ & $\begin{array}{c}0.109 \\
0.148(37)\end{array}$ & $\begin{array}{c}0.311 \\
0.314(26)\end{array}$ \\
\hline Mean ${ }^{1}$ & $\begin{array}{c}0.295 \\
0.297(26)\end{array}$ & $\begin{array}{c}0.238 \\
0.255(35)\end{array}$ & $\begin{array}{c}0.058 \\
0.095(38)\end{array}$ & $\begin{array}{c}0.376 \\
0.381(36)\end{array}$ & $\begin{array}{c}0.342 \\
-\end{array}$ & $\begin{array}{c}0.122 \\
0.160(37)\end{array}$ & $\begin{array}{c}0.315 \\
0.317(26)\end{array}$ \\
\hline $1^{3}$ & $\begin{array}{c}0.295 \\
0.297(34)\end{array}$ & $\begin{array}{c}0.000 \\
0.035(1)\end{array}$ & $\begin{array}{c}0.209 \\
0.258(1)\end{array}$ & $\begin{array}{c}0.176 \\
0.177(16)\end{array}$ & $\begin{array}{c}0.094 \\
0.097(15)\end{array}$ & $\begin{array}{c}0.110 \\
0.126(1)\end{array}$ & - \\
\hline
\end{tabular}

${ }^{1}$ Mean of the four different $k_{w a}$ or $k_{i n h}$ values, respectively, was used for modeling. ${ }^{2}$ Minimum of the four different $k_{w a}$ or $k_{i n h}$ values, respectively, was used for modeling (worst case). ${ }^{3}$ Factor set to 1.

In contrast to the differently treated materials, a good fit between relative $D_{R d}$ and $v_{\text {rel. }}$. was observed for the untreated timbers $\left(\mathrm{R}^{2}=0.823\right)$ at a threshold for $k_{i n h}$ and $k_{w a}$ of $T h r=3$. Meyer-Veltrup et al. [18] only used untreated wood for the initial model which had an even better fit $\left(R^{2}=0.912\right)$, but covered a smaller range of durability. In this study, several very durable tropical hardwood species were considered, such as Bongossi, Amaranth, merbau, Basralocus, and teak. Furthermore, a larger variety of data sources representing different test methods, test locations, and inspection and assessment techniques were considered and helped explain that both $D_{R d}$ and $v_{\text {rel }}$. scatter more compared to the previous model. However, the model was robust even when including several very durable wood species from a variety of data sources $\left(R^{2}=0.823\right)$.

\section{Conclusions}

The Meyer-Veltrup model predicts relative decay rates $\left(v_{\text {rel. }}\right)$ based on a material resistance dose representing the inherent protective properties of wood and its wetting ability. The optimized Meyer-Veltrup model presented in this paper produced reliable decay rate estimates for a range of new species and treatments. However, the predictive power of the model was stronger for untreated than for modified and preservative-treated wood. Both modified and treated wood add influence factors to the model, such as type and concentration of the preservative, varying levels of treatment intensity (e.g., weight present gain or treatment temperatures), process conditions, and an increased variability within and between treatment batches. It is assumed that material- and treatment-specific parameters of water-repellant treated and coated wood will also affect the reliability of the model. To further increase the accuracy and fit of the model, more data are needed, which should: (1) match laboratory indicators and field performance, and (2) cover further parameters, which are specific for treated wood and were lacking (i.e., unavailable) in the recent study.

Assessing data quality remains a major challenge when utilizing test data, especially for treated material. Due to the lack of directly matched data for a specific material, it is unavoidable to group sets of data that are similar but still show some discrepancies. In Part 3 of this paper [98], we will utilize the presented modeling approach and provide estimates of relative decay rates based on: (1) above-ground field tests at many different locations, and (2) relative $D_{R d}$ values from an intensive global survey on durability data. 
Author Contributions: G.A. and C.B. were mainly responsible for the conceptualization, methodology used, data evaluation, data validation, and formal analysis. Investigations and data curation were conducted by all authors. The original draft of this article was prepared by C.B. who was also responsible for the review and editing process of this article. C.B. provided the visualization. All authors have read and agreed to the published version of the manuscript.

Funding: G.A., C.B., S.F., and E.S. received funding in the frame of the research project CLICKdesign, which is supported under the umbrella of ERA-NET Cofund ForestValue by the Ministry of Education, Science and Sport (MIZS)—Slovenia; the Ministry of the Environment (YM)—Finland; the Forestry Commissioners (FC)—UK; Research Council of Norway (RCN, 297899)—Norway; the French Environment and Energy Management Agency (ADEME) and the French National Research Agency (ANR)-France; the Swedish Research Council for Environment, Agricultural Sciences and Spatial Planning (FORMAS), Swedish Energy Agency (SWEA), Swedish Governmental Agency for Innovation Systems (Vinnova)—Sweden; and the Federal Ministry of Food and Agriculture (BMEL) and Agency for Renewable Resources (FNR) - Germany. ForestValue has received funding from the European Union's Horizon 2020 research and innovation program under grant agreement $\mathrm{N}^{\circ} 773324$. We acknowledge support by the Open Access Publication Funds of the Goettingen University.

Institutional Review Board Statement: Not applicable.

Informed Consent Statement: Not applicable.

Data Availability Statement: The entire set of raw data presented in this study is available on request from the corresponding author.

Acknowledgments: The authors gratefully acknowledge Jonas Niklewski for technical advice on the suitability of data and models for implementation in existing service life prediction framework.

Conflicts of Interest: The authors declare no conflict of interest. The funders had no role in the design of the study; in the collection, analyses, or interpretation of data; in the writing of the manuscript, or in the decision to publish the results.

\section{References}

1. Leicester, R.H.; Wang, C.H.; Nguyen, M.N.; MacKenzie, C.E. Design of Exposed Timber Structures. Austr. J. Struct. Eng. 2009, 9, 241-248. [CrossRef]

2. Thelandersson, S.; Isaksson, T.; Suttie, E.; Frühwald, E.; Toratti, T.; Grüll, G.; Viitanen, H.; Jermer, J. Service Life of Wood in Outdoor above Ground Applications_Engineering Design Guideline. Background Document; Report TVBK-3061; Div. of Structural Engineering; Lund University: Lund, Sweden, 2011.

3. Isaksson, T.; Thelandersson, S.; Jermer, J.; Brischke, C. Beständighet för Utomhusträ Ovan Mark. Guide för Utformning och Materialval; Report TVBK-3066; Division of Structural Engineering; Lund University: Lund, Sweden, 2014.

4. Pousette, A.; Malo, K.A.; Thelandersson, S.; Fortino, S.; Salokangas, L.; Wacker, J. Durable Timber Bridges—Final Report and Guidelines; RISE Research Institutes of Sweden: Skellefteå, Sweden, 2017.

5. $\quad$ Blanchette, R.A.; Held, B.W.; Jurgens, J.A.; McNew, D.L.; Harrington, T.C.; Duncan, S.M.; Farrell, R.L. Wood-destroying soft rot fungi in the historic expedition huts of Antarctica. Appl. Environ. Microbiol. 2004, 70, 1328-1335. [CrossRef]

6. Sterflinger, K.; Tesei, D.; Zakharova, K. Fungi in hot and cold deserts with particular reference to microcolonial fungi. Fungal Ecol. 2012, 5, 453-462. [CrossRef]

7. EN 113-2. Durability of Wood and Wood-Based Products-Test Method against Wood Destroying Basidiomycetes-Part. 2: Assessment of Inherent or Enhanced Durability; European Committee for Standardization: Brussels, Belgium, 2020.

8. AWPA E10. Laboratory Method for Evaluating the Decay Resistance of Wood-Based Materials against Pure Basidiomycete Cultures: Soil/Block Test; American Wood Preservers' Association: Hoover, AL, USA, 2016.

9. AWPA E7. Standard Field Test for Evaluation of Wood Preservatives to Be Used in Ground Contact (UC4A, UC4B, UC4C); Stake Test; American Wood Preservers' Association: Hoover, AL, USA, 2015.

10. EN 252. Field Test Method for Determining the Relative Protective Effectiveness of a Wood Preservative in Ground Contact; European Committee for Standardization: Brussels, Belgium, 2015.

11. ENV 807. Wood Preservatives-Determination of the Effectiveness against Soft Rotting Micro-Fungi and Other Soil Inhabiting MicroOrganisms; European Committee for Standardization: Brussels, Belgium, 2001.

12. CEN/TS 15083-2. Durability of Wood and Wood-Based Products-Determination of the Natural Durability of Solid Wood against Wood-Destroying Fungi, Test Methods_Part. 2: Soft Rotting Micro-Fungi; European Committee for Standardization: Brussels, Belgium, 2015.

13. AWPA E14. Laboratory Method for Rapidly Evaluating the Decay Resistance of Wood-Based Materials in Ground Contact: Soil Bed Test; American Wood Preservers' Association: Hoover, AL, USA, 2016. 
14. Brischke, C.; Welzbacher, C.R.; Meyer, L.; Bornemann, T.; Larsson-Brelid, P.; Pilgård, A.; Frühwald Hansson, E.; Westin, M.; Rapp, A.O.; Thelandersson, S.; et al. Service Life Prediction of Wooden Components-Part 3: Approaching a Comprehensive Test Methodology. In Proceedings of the IRG Annual Meeting, IRG/WP 11-20464, Queenstown, New Zealand, 8-12 May 2011 ; p. 25.

15. Brischke, C.; Meyer, L.; Alfredsen, G.; Humar, M.; Francis, L.; Flæte, P.O.; Larsson-Brelid, P. Natural durability of timber exposed above ground-A survey. Drv. Ind. 2013, 64, 113-129. [CrossRef]

16. Meyer, L.; Brischke, C.; Preston, A. Testing the durability of timber above ground: A review on methodology. Wood Mater. Sci. Eng. 2016, 11, 283-304. [CrossRef]

17. EN 350. Durability of Wood and Wood-Based Products-Testing and Classification of the Durability to Biological Agents of Wood and Wood-Based Materials; European Committee for Standardization: Brussels, Belgium, 2016.

18. Meyer-Veltrup, L.; Brischke, C.; Alfredsen, G.; Humar, M.; Flæte, P.O.; Isaksson, T.; Larsson Brelid, P.; Westin, M.; Jermer, J. The combined effect of wetting ability and durability on outdoor performance of wood: Development and verification of a new prediction approach. Wood Sci. Technol. 2017, 51, 615-637. [CrossRef]

19. Humar, M.; Kržišnik, D.; Lesar, B.; Brischke, C. The performance of wood decking after five years of exposure: Verification of the combined effect of wetting ability and durability. Forests 2019, 10, 903. [CrossRef]

20. Kržišnik, D.; Brischke, C.; Lesar, B.; Thaler, N.; Humar, M. Performance of wood in the Franja partisan hospital. Wood Mater. Sci. Eng. 2019, 14, 24-32. [CrossRef]

21. Brischke, C.; Hesse, C.; Meyer-Veltrup, L.; Humar, M. Studies on the material resistance and moisture dynamics of Common juniper, yew, black cherry, and rowan. Wood Mater. Sci. Eng. 2018, 13, 222-230. [CrossRef]

22. De Angelis, M.; Romagnoli, M.; Vek, V.; Poljanšek, I.; Oven, P.; Thaler, N.; Lesar, B.; Kržišnik, D.; Humar, M. Chemical composition and resistance of Italian stone pine (Pinus pinea L.) wood against fungal decay and wetting. Ind. Crops Prod. 2018, 117, 187-196. [CrossRef]

23. Humar, M.; Balzano, A.; Grbec, S.; Gričar, J.; Kržišnik, D.; Lesar, B.; Vek, V. Investigation of the material resistance and moisture performance of pubescent oak (Quercus pubescens). Holzforschung 2021, 75, 22-36. [CrossRef]

24. Emmerich, L.; Militz, H.; Brischke, C. Long-term performance of DMDHEU-treated wood installed in different test set-ups in ground, above ground and in the marine environment. Int. Wood Prod. J. 2020, 11, 27-37. [CrossRef]

25. Meyer-Veltrup, L.; Brischke, C.; Niklewski, J.; Frühwald Hansson, E. Design and performance prediction of timber bridges based on a factorization approach. Wood Mater. Sci. Eng. 2018, 13, 167-173. [CrossRef]

26. Brischke, C.; Humar, M.; Niklewski, J.; Alfredsen, G. Modelle zur Vorhersage der Gebrauchsdauer von Holzbauteilen auf dem Prüfstand. Holztechnologie 2019, 60, 23-34.

27. Cook, J.A.; Morris, P.I. Modeling data from stake tests of waterborne wood preservatives. For. Prod. J. 1995, 45, 61.

28. Isaksson, T.; Brischke, C.; Thelandersson, S. Development of decay performance models for outdoor timber structures. Mater. Struct. 2013, 46, 1209-1225. [CrossRef]

29. Brischke, C. Untersuchung Abbaubestimmender Faktoren zur Vorhersage der Gebrauchsdauer Feuchtebeanspruchter Holzbauteile. Doctoral's Thesis, University of Hamburg, Hamburg, Germany, 2007.

30. Findlay, W.P.K. The natural resistance to decay of some Empire timbers. Emp. For. J. 1938, 17, $249-259$.

31. Van Acker, J.; De Windt, I.; Li, W.; Van den Bulcke, J. Critical parameters on moisture dynamics in relation to time of wetness as factor in service life prediction. In Proceedings of the IRG Annual Meeting, IRG/WP/14-20555, St. George, UT, USA, 11-15 May 2014 ; p. 22.

32. Westin, M. Durability of furfurylated wood-Results from laboratory and field tests in the Ecobinders project. In Proceedings of the IRG Annual Meeting, IRG/WP/12-40602, Kuala Lumpur, Malaysia, 6-10 May 2011; p. 7.

33. Westin, M.; Conti, E.; Creemers, J.; Flæte, P.O.; Gellerich, A.; Irbe, I.; Klamer, M.; Melcher, E.; Moeller, R.; Nunes, L.; et al. 10 year Report on COST E37 Round Robin Tests-Comparison of results from laboratory and field tests. In Proceedings of the IRG Annual Meeting, IRG/WP/17-30718, Ghent, Belgium, 4-8 June 2017; p. 13.

34. Emmerich, L.; Brischke, C.; Sievert, M.; Schulz, M.S.; Jaeger, A.C.; Beulshausen, A.; Humar, M. Predicting the Outdoor Moisture Performance of Wood Based on Laboratory Indicators. Forests 2020, 11, 1001. [CrossRef]

35. Wälchli, O. Die Widerstandsfähigkeit verschiedener Holzarten gegen Angriffe durch den echten Hausschwamm (Merulius lacrimans (Wulf.) Fr.). Holz Roh Werkst. 1973, 31, 96-102. [CrossRef]

36. Wälchli, O. Die Widerstandsfähigkeit verschiedener Holzarten gegen Angriffe durch Coniophora puteana (Schum. ex Fr.) Karst. (Kellerschwamm) und Gloephyllum trabeum (Pers. ex Fr.) Murrill (Balkenblättling). Holz Roh Werkst. 1976, 34, 335-338. [CrossRef]

37. Clark, J.W. Natural Decay Resistance of Fifteen Exotic Woods Imported for Exterior Use; U.S. Forest Service, Forest Products Laboratory Research Paper, FPL-RP-103; United States Department of Agriculture: Washington, DC, USA, 1969.

38. Bultman, J.D.; Southwell, C.R. Natural Resistance of Tropical American Woods to Terrestrial Wood-Destroying Organisms. Biotropica 1976, 8, 71-95. [CrossRef]

39. Van Acker, J.; Stevens, M.; Carey, J.; Sierra-Alvarez, R.; Militz, H.; Le Bayon, I.; Kleist, G.; Peek, R.D. Biological durability of wood in relation to end-use. Holz Roh Werkst. 2003, 61, 35-45. [CrossRef]

40. Van Acker, J.; Militz, H.; Stevens, M. The significance of accelerated laboratory testing methods determining the natural durability of wood. Holzforschung 1999, 53, 449-458. [CrossRef] 
41. Edlund, M.-L. Durability of Some Alternatives to Preservative-Treated Wood. In Proceedings of the IRG Annual Meeting, IRG/WP 04-30353, Ljubljana, Slovenia, 6-10 June 2004; p. 13.

42. Van den Bulcke, J.; De Windt, I.; Defoirdt, N.; Van Acker, J. Non-Destructive Evaluation of Wood Decay. In Proceedings of the IRG Annual Meeting, IRG/WP/11-20479, Queenstown, New Zealand, 8-12 May 2011; p. 11.

43. Brischke, C.; Meyer, L.; Olberding, S. Durability of wood exposed in ground-Comparative field trials with different soil substrates. Int. Biodeterior. Biodegrad. 2014, 86, 108-114. [CrossRef]

44. Brischke, C.; Welzbacher, C.R.; Gellerich, A.; Bollmus, S.; Humar, M.; Plaschkies, K.; Scheiding, W.; Alfredsen, G.; Van Acker, J.; De Windt, I. Wood natural durability testing under laboratory conditions: Results from a round-robin test. Eur. J. Wood Wood Prod. 2014, 72, 129-133. [CrossRef]

45. Brischke, C.; Gellerich, A.; Militz, H.; Starck, M. Performance of coated and uncoated horizontal lap-joint members during 20 years of outdoor exposure. Wood Res. 2017, 62, 883-894.

46. Meyer, L.; Brischke, C.; Melcher, E.; Brandt, K.; Lenz, M.T.; Soetbeer, A. Durability of English oak (Quercus robur L.)—Comparison of decay progress and resistance under various laboratory and field conditions. Int. Biodeter. Biodegr. 2014, 86, 79-85. [CrossRef]

47. Thaler, N.; Žlahtič, M.; Humar, M. Performance of recent and old sweet chestnut (Castanea sativa) wood. Int. Biodeterior. Biodegrad. 2014, 94, 141-145. [CrossRef]

48. Ugovšek, A.; Šubic, B.; Starman, J.; Rep, G.; Humar, M.; Lesar, B.; Thaler, N.; Brischke, C.; Meyer-Veltrup, L.; Jones, D.; et al. Short-term performance of wooden windows and facade elements made of thermally modified and non-modified Norway spruce in different natural environments. Wood Mater. Sci. Eng. 2019, 14, 42-47. [CrossRef]

49. Scheiding, W.; Jacobs, K.; Bollmus, S.; Brischke, C. Durability Classification of Treated and Modified Wood-Approaching a Guideline for Sampling, Testing, and Statistical Analysis. In Proceedings of the IRG Annual Meeting, IRG/WP 20-20676, Webinar, 10-11 June 2020; p. 8.

50. Cookson, L.J. Determining the Natural Durability of Eucalypts in Australia. In Durable Eucalypts on Drylands: Protecting and Enhancing Value; Altaner, C.M., Murray, T.J., Morgenroth, J., Eds.; Marlborough Research Centre: Blenheim, New Zealand, 2017; pp. 77-84.

51. Reinprecht, L.; Vidholdová, Z. Rot resistance of tropical wood species affected by water leaching. BioResources 2019, 14, 8664-8677.

52. Deklerck, V.; De Ligne, L.; Espinoza, E.; Beeckman, H.; Van den Bulcke, J.; Van Acker, J. Assessing the natural durability of xylarium specimens: Mini-block testing and chemical fingerprinting for small-sized samples. Wood Sci. Technol. 2020, 54, 981-1000. [CrossRef]

53. Deklerck, V.; De Windt, I.; Defoirdt, N.; Van den Bulcke, J.; Beeckman, H.; Espinoza, E.; Van Acker, J. Assessing the Natural Durability for Different Tropical Timber Species Using the Mini-Block Test. In Proceedings of the IRG Annual Meeting, IRG/WP 17-10886, Ghent, Belgium, 4-8 June 2017; p. 14.

54. Seehann, G. Zur natürlichen Dauerhaftigkeit von Kempas und Keruing gegenüber holzzerstörenden Pilzen. Holz Roh Werkst. 1973, 31, 269-272. [CrossRef]

55. Smith, G.A.; Orsler, R.J. The biological natural durability of timber in ground contact. In Proceedings of the IRG Annual Meeting, IRG/WP 94-20051, Nusa Dua, Bali, Indonesia, 29 May-3 June 1994; p. 23.

56. Welzbacher, C.R.; Rapp, A.O. Determination of the water sorption properties and preliminary results from field tests above ground of thermally modified material from industrial scale processes. In Proceedings of the IRG Annual Meeting, IRG/WP/04-40279, Ljubljana, Slovenia, 6-10 June 2004; p. 14.

57. Augusta, U. Untersuchung der Natürlichen Dauerhaftigkeit Wirtschaftlich Bedeutender Holzarten bei Verschiedener Beanspruchung im Außenbereich. Doctoral's Thesis, University of Hamburg, Hamburg, Germany, 2007.

58. Conti, E. English Oak-Natural Durable Timber-Laboratory Test Results. IRG/WP Durability Database; The International Research Group on Wood Protection, IRG/WP/DDB 14-00014; IRG Secretariat: Stockholm, Sweden, 2014.

59. Brischke, C.; Meyer-Veltrup, L. Performance of thermally modified wood during 14 years of outdoor exposure. Int. Wood Prod. J. 2016, 7, 89-95. [CrossRef]

60. Hundhausen, U.; Flæte, P.O.; Mahnert, K.C.; Bysheim, K. Overflatekvalitet på terrassematerialer-Resultater etter to års eksponering. Tretek. Inf. 2016, 1, 23-27.

61. Westin, M.; Alfredsen, G. Durability of Modified Wood in UC3 and UC4-Results from Lab Tests and 5 Years Testing in 3 Fields. In Proceedings of the IRG Annual Meeting, IRG/WP/11-40562, Queenstown, New Zealand, 8-12 May 2011 ; p. 9.

62. Bavendamm, W.; Anuwongse, B. Über die Fäulnisresistenz thailändischer Holzarten. Holz Roh Werkst. 1967, $25,392-393$. [CrossRef]

63. Francis, L.P.; Norton, J.; Melcher, E.; Wong, A.H.H.; Kok Lai, J.; Klamer, M.; Konkler, M.J.; Morrell, J.J. Performance of Untreated Timbers in above Ground Decking Tests: Preliminary Results from an International Collaborative Trial. In Proceedings of the IRG Annual Meeting, IRG/WP 19-10940, Quebec City, QC, Canada, 12-16 May 2019; p. 21.

64. Petrenko, I.A. Stoikost' Zaboloni I Yadra Listvennitsy Sibirskoi K Porazheniyu Razlichnymi Vidami Domovykh Gribov; Sibirskii Tekhnologicheskii Institute: Krasnoyarsk, Russia, 1964; pp. 261-264. (In Russian)

65. Venäläinen, M.; Heikkonen, S.; Terziev, N.; Torniainen, P. Durability of the Siberian larch heartwood timber of different origin: The results of 11-year ground contact test in Finland. Sib. J. For. Sci. 2019, 3, 14-19, (in English with Russian abstract).

66. Highley, T.L. Comparative Durability of Untreated Wood in Use Above Ground. Int. Biodeterior. Biodegrad. 1995, 35, 409-419. [CrossRef] 
67. Meyer, L.; Brischke, C.; Pilgård, A. Moisture Performance Based Wood Durability Testing. In Proceedings of the IRG Annual Meeting, IRG/WP 12-20495, Kuala Lumpur, Malaysia, 6-10 May 2012; p. 26.

68. Metsä Kortelainen, S.; Viitanen, H. Durability of thermally modified sapwood and heartwood of Scots pine and Norway spruce in the modified double layer test. Wood Mater. Sci. Eng. 2017, 12, 129-139. [CrossRef]

69. Brischke, C.; Melcher, E. Performance of wax-impregnated timber out of ground contact: Results from long-term field testing. Wood Sci. Technol. 2015, 49, 189-204. [CrossRef]

70. Schulz, G. Vergleichende Untersuchungen mit verschiedenen Stämmen von Lentinus lepideus, gleichzeitig ein Beitrag zum Soil-Block-Verfahren. Holz Roh Werkst. 1958, 16, 435-444. [CrossRef]

71. Morris, P.I.; Ingram, J.; Larkin, G.; Laks, P. Field tests of naturally durable species. For. Prod. J. 2011, 61, 344-351. [CrossRef]

72. Palanti, S. Evaluation of Durability Conferred by an Oleothermic Treatment on Chestnut and Douglas fir through Laboratory and in Field Tests. Open J. For. 2013, 3, 66-69.

73. Stirling, R.; Wong, D. Performance of Naturally Durable Decks after 15 Years of Field Exposure. In Proceedings of the IRG Annual Meeting, IRG/WP 20-10963, Webinar, 10-11 June 2020; p. 9.

74. Plaschkies, K.; Scheiding, W.; Jacobs, K.; Rangno, N. Virulence of two Laboratory Test Strains and one Natural Isolate of Rhodonia (Oligoporus) placenta against Thermally Modified Pine and Beech Wood. In Proceedings of the IRG Annual Meeting, IRG/WP 13-20524, Stockholm, Sweden, 16-20 June 2013; p. 9.

75. Viitanen, H.; Metsä-Kortelainen, S. Testing of decay resistance of sapwood and heartwood of thermally modified Scots pine and Norway spruce. In Proceedings of the IRG Annual Meeting, IRG/WP/10-40523, Biarritz, France, 9-13 May 2010 ; p. 10.

76. Alfredsen, G.; Brischke, C.; Meyer-Veltrup, L.; Humar, M.; Flæte, P.-O. The effect of different test methods on durability classification on modified wood. ProLigno 2017, 13, 290-297.

77. Jacobs, K.; Scheiding, W.; Weiß, B. Durability of acetylated Radiata pine: Laboratory tests and performance in practice. In Proceedings of the IRG Annual Meeting, IRG/WP 20-40899, Webinar, 10-11 June 2020; p. 14.

78. Ziethén, R.; Brynildsen, P.; Lande, S.; Kristoffersen, J.; Westin, M. Kebony-An Alternative to Teak for Boat Decking. In Proceedings of the Fourth European Conference on Wood Modification, Norra Latin City Conference Centre, Stockholm, Sweden, 27-29 April 2009; SP Technical Research Institute of Sweden: Boras, Sweden, 2009.

79. Alfredsen, G.; Flæte, P.O.; Militz, H. Performance of Novel Wood Protection Systems-Evaluation Based on Five Different Test Setups. In Proceedings of the International Convention of Society of Wood Science and Technology and United Nations Economic Commission for Europe-Timber Committee, Geneva, Switzerland, 11-14 October 2010.

80. Emmerich, L.; Ehrmann, A.; Brischke, C.; Militz, H. Comparative studies on the durability and moisture performance of wood modified with cyclic N-methylol and N-methyl compounds. Wood Sci. Technol. 2021, submitted.

81. Humar, M.; Lesar, B.; Thaler, N. Performance of Copper Treated and Naturally Durable Wood in Laboratory and Outdoor Conditions. In Proceedings of the International Conference on Durability of Building Materials and Components, Sao Paulo, Brazil, 2-5 September 2014; pp. 722-727.

82. Humar, M.; Lesar, B.; Thaler, N.; Kržišnik, D.; Žlatič, M. Influence of the Retention and Penetration of Cu Based Preservatives on the Performance of Softwoods in Ground. In Designing with Bio-Based Building Materials—Challenges and Opportunities, Book of Abstract from Joint Technical Workshop, Madrid, Spain, 24-25 February 2016; De Troya, T., Ed.; National Institute for Agricultural and Food Research and Technology: Madrid, Spain; Eduardo Torroja Institute for Construction Sciences: Madrid, Spain, 2016; pp. 61-62.

83. Alfredsen, G.; Brischke, C.; Marais, B.N.; Stein, R.F.A.; Zimmer, K.; Humar, M. Modelling the material resistance of wood-Part 1: Utilizing durability test data based on different reference wood species. Forests, 2021, 12, 558. [CrossRef]

84. Bravery, A.F. A Miniaturised Wood-Block Test for the Rapid Evaluation of Preservative Fungicides. In Proceedings of the A Special Seminar Held in Association with the 10th Annual Meeting of the IRG, Peebles, Scotland, 18-22 September 1978; Rep. No. 136; Swedish Wood Preservation Institute: Stockholm, Sweden, 1979.

85. CEN/TS 16818. Durability of Wood and Wood-Based Products-Moisture Dynamics of Wood and Wood-Based Products; European Committee for Standardization: Brussels, Belgium, 2018.

86. CEN/TS 12037. Wood Preservatives_Field Test Method for Determining the Relative Protective Effectiveness of a Wood Preservative Exposed out of Ground Contact_-Horizontal Lap-Joint Method; European Committee for Standardization: Brussels, Belgium, 2003.

87. EN 330. Wood Preservatives-Determination of the Relative Protective Effectiveness of a Wood Preservative for Use Under a Coating and Exposed out-of-Ground Contact_Field Test: L-Joint Method; European Committee for Standardization: Brussels, Belgium, 2015.

88. Stirling, R.; Alfredsen, G.; Brischke, C.; De Windt, I.; Francis, L.P.; Frühwald Hansson, E.; Humar, M.; Jermer, J.; Klamer, M.; Kutnik, M.; et al. Global survey on durability variation-On the effect of the reference species. In Proceedings of the IRG Annual Meeting, IRG/WP 16-20573, Lisbon, Portugal, 15-19 May 2016; p. 26.

89. Scheffer, T.C.; Morrell, J.J. Natural Durability of Wood: A Worldwide Checklist of Species; Forestry Publications Office; Oregon State University: Corvallis, OR, USA, 1998.

90. Shida, S.; Saito, Y. Effects of heat treatment on brittleness of Styrax tonkinensis wood. J. Wood Sci. 2007, 53, 181-186.

91. Brischke, C.; Welzbacher, C.R.; Rapp, A.O. Detection of fungal decay by high-energy multiple impact (HEMI) testing. Holzforschung 2006, 60, 217-222. [CrossRef]

92. Zelinka, S.L.; Glass, S.V. Water vapor sorption isotherms for southern pine treated with several waterborne preservatives. J. Test. Eval. 2010, 38, 521-525. 
93. Barney, R.; Carroll, J.; Delaet, D. Surfactant studies of quaternary ammonium compounds: Critical surfactant concentration. J. Surfact. Deterg. 2006, 9, 137-140. [CrossRef]

94. Kamdem, D.P. Copper-based systems for exterior residential applications. Am. Chem. Soc. (ACS) Symp. Ser. 2008, 982, 427-439.

95. Choi, S.; Ruddick, J.N.; Morris, P. Chemical redistribution in CCA-treated decking. For. Prod. J. 2004, 54, 33-37.

96. Stirling, R.; Ruddick, J.N.; Xue, W.; Morris, P.I.; Kennepohl, P. Characterization of copper in leachates from ACQ- and MCQ-treated wood and its effect on basidiospore germination. Wood Fib. Sci. 2015, 47, 209-216.

97. Thaler, N.; Humar, M. Copper leaching from copper-ethanolamine treated wood: Comparison of field test studies and laboratory standard procedures. Bioresources 2014, 9, 3038-3051. [CrossRef]

98. Brischke, C.; Alfredsen, G.; Humar, M.; Conti, E.; Cookson, L.; Emmerich, E.; Flæte, P.O.; Fortino, S.; Francis, L.; Hundhausen, U.; et al. Modelling the material resistance of wood-Part 3: Relative resistance in above and in ground situations-Results of a global survey. Forests 2021, submitted. 